\title{
ANASTOMOSES BETWEEN INFRAORBITAL ARTERY AND OPHTHALMIC ARTERY WITHIN THE ORBIT
}

\author{
Ye, Jingyang. Winters, Jacob *. Balsiger, Heather. Gest, Thomas. \\ Department of Medical Education, Texas Tech Health Sciences Center - El Paso Paul L. Foster \\ School of Medicine, El Paso, Texas 79905, USA.
}

\section{ABSTRACT}

Documentation of contributions from infraorbital artery to branches of the ophthalmic artery are lacking with respect to the orbit. While there have been sources that suggest anastomoses between the infraorbital artery and ophthalmic artery within the orbit, there has been no clear consensus on the location and significance of these anastomoses. This study aimed to identify and track the course of the infraorbital artery within the orbit and its possible anastomoses and supply to the orbit. Cadaveric analysis of 19 individuals (38 orbits) revealed a common pattern of anastomosis between the infraorbital and inferior palpebral arteries (95\%; $89 \%$ bilaterally) as well as course to the lacrimal sac (91\%; $73 \%$ bilaterally). These findings demonstrate the significance of infraorbital artery supply with respect to the inferior orbit as well as the lacrimal sac, which can prove clinically useful, such as in the context of surgical repair of orbital trauma.

KEY WORDS: Infraorbital Artery, Ophthalmic Artery, Palpebral Arteries, Orbital Vessels, Lacrimal Sac, Orbital Anastomoses.

Address for Correspondence: Jacob Winters, c/o Heather Balsiger, Department of Medical Education, Texas Tech Health Sciences Center El Paso Paul L. Foster School of Medicine, El Paso, Texas 79905, USA. E-Mail: jacob.winters@ttuhsc.edu

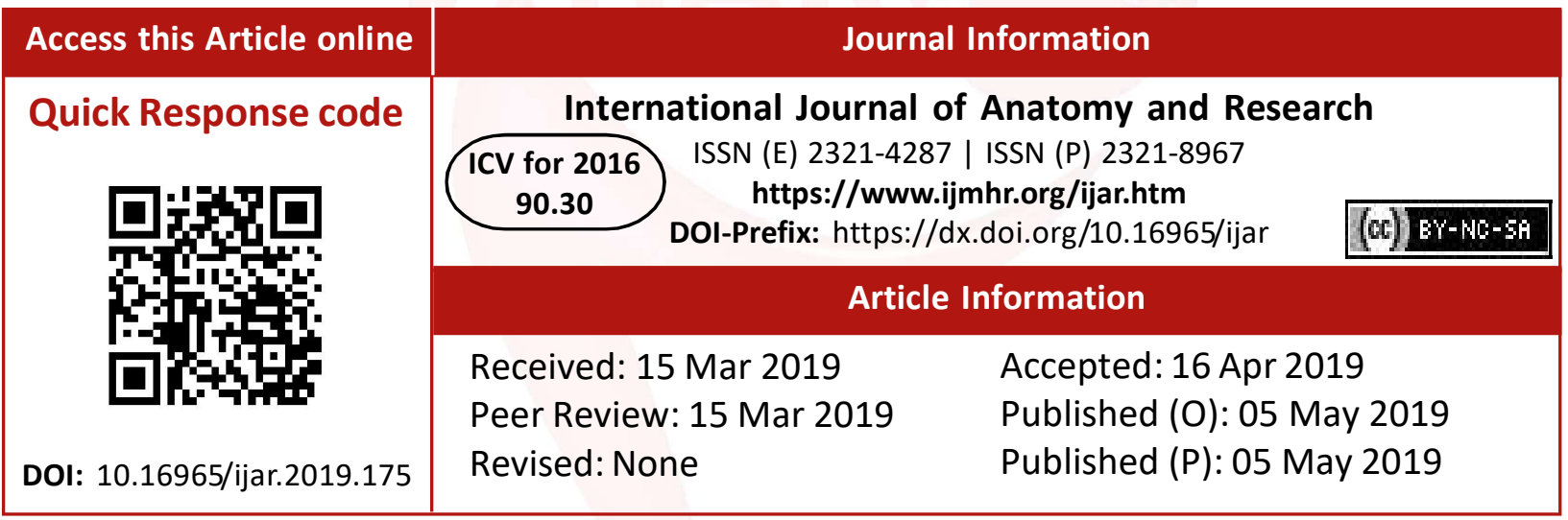

\section{INTRODUCTION}

The vasculature of the orbit has been well studied, and many variations of the vasculature have been noted. Anastomoses involving the ophthalmic artery are particularly important, given the relevance of the ophthalmic artery to the orbit. Extraorbital and deeper anastomoses have been documented [1]. However, most current texts do not note intraorbital anastomoses, particularly with respect to infraorbital artery. Although it has been noted that infraorbital artery may supply the lower orbit [2], attempts to elucidate the frequency and nature of these connections are sparse. A thorough understanding of the orbital network between the infraorbital artery and the ophthalmic artery is necessary, given the importance and risk related to surgical intervention in the orbit [3].

This study aimed to track the infraorbital artery and locate possible intraorbital anastomoses between infraorbital artery and branches of the ophthalmic artery. Previous studies of anastomoses of the orbit have noted an increased frequency of anastomosis in the right side [4]. With this in mind, this study will also look to track the laterality of intraorbital anastomoses between infraorbital artery and ophthalmic artery. To elucidate this, orbits from 19 individuals were dissected and analyzed, tracking the infraorbital artery from its origin in the orbital 
floor superiorly to possible anastomoses, documenting the frequency on each side, and noting any other novel courses of blood supply in the lower orbit.

\section{MATERIALS AND METHODS}

A total of 38 orbits from 19 cadavers (left and right orbits) were dissected and examined in the Texas Tech Health Sciences Center El Paso anatomy lab. The subjects ranged from 52 to 95 years old. Preliminary dissection of the orbits had been previously performed, removing the zygomatic bone at the lateral and inferior orbital walls to expose the orbital floor. Further dissection of the zygomatic and maxillary bones was performed to completely remove the orbital floor and to expose the lacrimal sacs medially.

From the orbital floor, the infraorbital artery was specifically located and tracked to find its course superiorly and subsequent anastomoses. Emphasis was put on locating possible anastomoses with branches of the ophthalmic artery, as well as tracking possible infraorbital courses that may supply the lacrimal sac. Of the 19 subjects, only 11 allowed viewing of the lacrimal sac area. Two of the subjects had previous orbital surgery, and as a result, the orbital floor and medial wall was covered in a mesh that obliterated some of the vessels for viewing. Six of the other subjects were merely orbital specimens with the medial aspects removed, including the lacrimal sac.

\section{RESULTS AND DISCUSSION}

Patterns of Anastomosis: Nearly all of the subjects showed anastomoses between infraorbital artery and the inferior palpebral arteries that arise from ophthalmic artery. Many of the subjects had branches of the infraorbital artery arise through the orbital floor to run superiorly and anastomose with both the medial and lateral inferior palpebral arteries. Of the 19 subjects, 18 exhibited anastomoses between infraorbital and inferior palpebral artery branches, with 17 exhibiting bilateral patterns of anastomosis. The most common pattern discovered was a branch of the infraorbital artery running superiorly, then splitting bilaterally to anastomose with both inferior palpebral arteries (Figure 1).
This pattern was seen in 10 of the 19 subjects bilaterally, 3 subjects in the left orbit, and 1 subject in the right orbit. Other patterns seen include anastomosing with the palpebral artery network without splitting bilaterally (seen in 2 bilaterally and 1 right orbit), an anastomosis medially with the medial inferior palpebral artery (seen in 1 right orbit), and an anastomosis laterally with the lateral inferior palpebral artery (seen in 1 right orbit). The 2 subjects who had previous orbital surgery showed infraorbitalpalpebral anastomoses, but the exact pattern was impossible to determine due to obliteration of small vessels in the area. One subject lacked any anastomoses on either side (Figure 2), and one subject showed anastomosis on only the right side. Of the total 19 subjects, 17 exhibited bilateral patterns of anastomosis (89\%), 17 exhibited left orbital anastomosis (89\%), and 18 exhibited right orbital anastomosis (95\%).

Fig. 1: The most common pattern seen in infraorbitalpalpebral anastomosis. The infraorbital artery runs first superiorly to the inferior eyelid, the splits medially and laterally (arrow) to anastomose with the lateral and medial inferior palpebral arteries. A branch can also be seen running to the lacrimal sac.

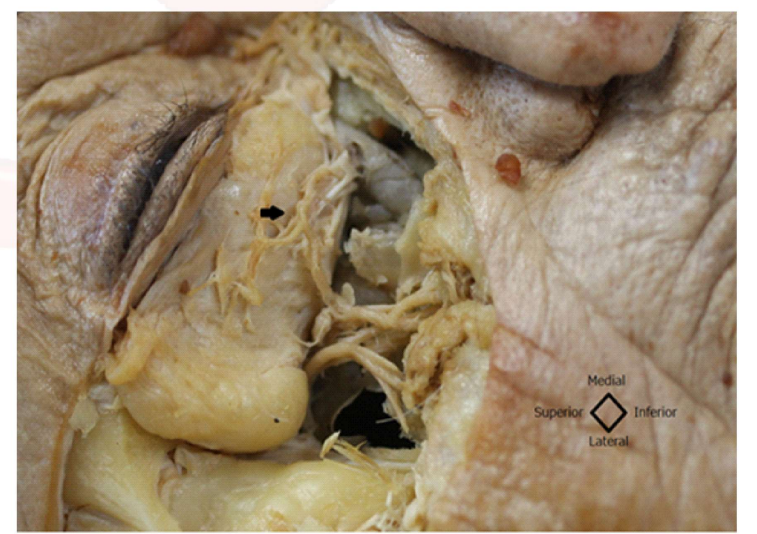

Fig. 2: Left orbit showing no anastomosis between infraorbital artery and inferior palpebral arteries. In this figure, the infraorbital neurovascular bundle can be seen running inferomedially to the facial artery.

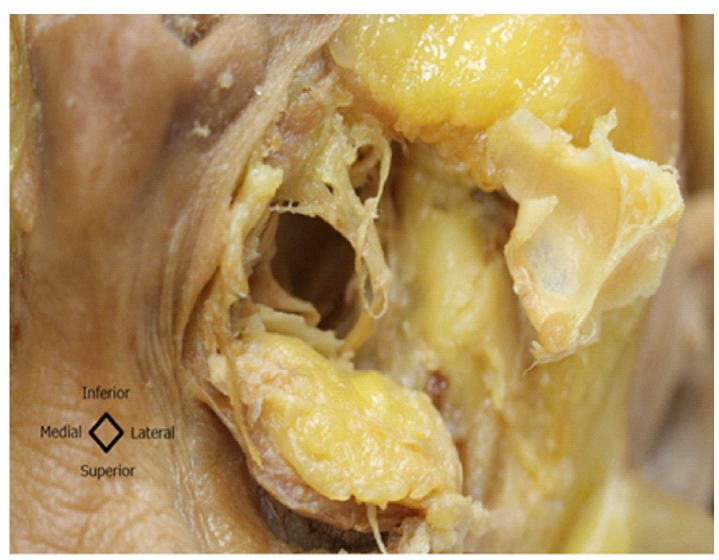


Lacrimal Sac Supply: Out of the 19 total subjects, 11 had intact lacrimal sac regions that could be exposed for arterial supply. Of the 11, 8 subjects had a viewable branch from the infraorbital-inferior palpebral anastomosis that ran medially to the lacrimal sac in the left orbit (73\%), 10 in the right orbit (91\%), and 8 bilaterally (73\%). This pattern can be seen in Figure 1.

Analysis by Sex: Sex could be determined in 13 of the 19 subjects, with four males and nine females. Three of the males (75\%) exhibited bilateral patterns of anastomosis between infraorbital and inferior palpebral arteries, with all four males having a right orbital anastomosis (100\%). A similar pattern was seen for lacrimal sac supply, where three males had a branch running to the lacrimal sac in the left orbit (75\%) and all four having a branch to the lacrimal sac in the right orbit (100\%). In the females, eight of the nine $(89 \%)$ subjects showed bilateral anastomoses, with one having absent anastomoses on either side. Of the seven females with observable lacrimal sacs, five had supply from the infraorbital-inferior palpebral anastomosis in the left orbit (71\%), and six had supply in the right orbit (86\%).

A summary of the results can be seen in Tab.1. Table 1: Indicates presence of anastomosis between infraorbital and inferior palpebral arteries in each orbital, as well as blood supply to the lacrimal sacs.

\begin{tabular}{|c|c|c|c|c|}
\hline Subjects & Lt Anas. & Rt Anas. & Lt Lac. Sac & Rt Lac. Sac \\
\hline Total & 17 & 18 & 8 & 10 \\
\hline Total Subjects & 19 & 19 & 11 & 11 \\
\hline Fraction & 0.895 & 0.947 & 0.727 & 0.909 \\
\hline & & & & \\
\hline Male Total & 3 & 4 & 3 & 4 \\
\hline Male Total Subjects & 4 & 4 & 4 & 4 \\
\hline Male Fraction & 0.75 & 1 & 0.75 & 1 \\
\hline & & & & \\
\hline Female Total & 8 & 8 & 5 & 6 \\
\hline Female Total Subjects & 9 & 9 & 7 & 7 \\
\hline Female Fraction & 0.889 & 0.889 & 0.714 & 0.857 \\
\hline
\end{tabular}

\section{DISCUSSION}

The results of this study confirmed the anastomosis between infraorbital artery and the inferior palpebral arteries that had been suggested in other studies. This study also shows the less documented course from the infraorbital-inferior palpebral anastomosis to the lacrimal sac and demonstrates a potentially important source of blood supply for the lacrimal sac. A large majority of subjects (95\%) were discovered to have some pattern of anastomosis between the infraorbital artery and the inferior palpebral arteries. A bilateral pattern of anastomosis was common, found in $89 \%$ of the subjects. A complete lack of this pattern of anastomosis was found in only one subject, and only one other subject lacked this pattern of anastomosis unilaterally. Results show a slight tendency for right sided infraorbital-palpebral anastomosis, but given the small sample size and difference of one subject, further studies will have to be done to confirm possible lateralization tendencies of anastomoses.

Lacrimal sac supply was also found in a significant majority of the subjects. In the 11 subjects, $73 \%$ exhibited bilateral supply from the infraorbital-inferior palpebral anastomosis. Right sided lateralization of lacrimal sac supply was more prominent than that found in patterns of anastomosis, with $91 \%$ of the subjects' exhibiting a branch to the lacrimal sac in the right orbit. Again, however, the small sample size of 11 must be considered.

Analysis by sex showed little deviation from the overall results. Males were found to have a slightly lower rate of left sided anastomosis (75\%) and slightly higher rate of right-sided anastomosis (100\%). However, these results should be considered in the context of the sample size of 4. Comparatively, females exhibited closer results to the average.

\section{CONCLUSION}

This study attempted to find patterns of anastomosis between the ophthalmic artery and infraorbital artery within the orbit. The findings confirmed the existence of an anastomosis between the infraorbital artery and the inferior palpebral arteries as well as branches of the ophthalmic artery as described by Hayreh [2]. Contrary to many texts that do not show this anastomosis, the results show that it is a rather common pattern, as it was found in a large majority of the subjects bilaterally. The results of this study also support a previous study that found increased frequency of anastomoses in right sided orbits [4], although given the small sample size of this study $(n=19)$, its findings should not be taken to be conclusive. A course running from the infraorbital-inferior palpebral 
anastomosis to the lacrimal sac was also found to be prevalent in the subjects of this study, which supports claims made in previous studies [2].

This study's findings provide clearer understanding in the vasculature of the inferior orbit, demonstrating the infraorbital artery's role in supply to the inferior palpebrae, orbital floor, and lacrimal sac medially. These findings have significance clinically given the importance of understanding the blood supply of the region during surgical intervention to avoid potential complications [3].

Further investigation can be done in patterns of infraorbital artery branches arising through the orbital floor. During this study, a number of the subjects exhibited different patterns of infraorbital artery penetration of the floor, with some rising laterally, medially, or bilaterally within the orbit. A clearer understanding of possible patterns and frequencies of these courses would further benefit the field of surgical intervention.

\section{ACKNOWLEDGEMENTS}

The authors would like to thank Amanda Herrera and Faustino Salcido for their contributions to this study. Additionally, the authors would like to thank the donors, without whose sacrifice this research would not be possible.

\section{Conflicts of Interests: None}

\section{REFERENCES}

[1]. Michalinos A, Zogana S, Kotsiomitis E, Mazarakis A, Troupis T. Anatomy of the ophthalmic artery: A review concerning its modern surgical and clinical applications. Anatomy Research International 2015; 2015:Article ID 591961 (8 pages).

[2]. Hayreh SS. Orbital vascular anatomy. Eye 2006;20:1130-1144.

[3]. Geibprasert S, Pongpech S, Armstrong D, Krings T. Dangerous extracranial-intracranial anastomoses and supply to the cranial nerves: Vessels the neurointerventionalist needs to know. AJNR Am J Neuroradiol 2009;30(8):1459-1468.

[4]. Berthelot JL, Hureau J. Clinical anatomical study of the macroscopic anastomoses of the ophthalmic artery in the periorbital region. J Anat Clin 1982;3:271.

How to cite this article:

Ye, Jingyang. Winters, Jacob. Balsiger, Heather. Gest, Thomas. ANASTOMOSES BETWEEN INFRAORBITAL ARTERY AND OPHTHALMIC ARTERY WITHIN THE ORBIT. Int J Anat Res 2019;7(2.2):6590-6593. DOI: 10.16965/ijar.2019.175 